\title{
Surgery for shoulder impingement: a systematic review and meta-analysis of controlled clinical trials
}

\author{
Moin Khan MD MSc, Bashar Alolabi MD MSc, Nolan Horner MD, Asheesh Bedi MD, \\ Olufemi R. Ayeni MD PhD, Mohit Bhandari MD PhD
}

\section{Abstract}

Background: Shoulder impingement is one of the most common nontraumatic upper limb causes of disability in adults. Our aim was to evaluate the efficacy of surgical intervention in comparison with nonoperative or sham treatments in patients with shoulder impingement in terms of both pain and functional outcomes.

Methods: We conducted a systematic review and meta-analysis of randomized controlled trials. Two reviewers independently screened MEDLINE, Embase, PubMed and Cochrane databases for randomized controlled trials published from 1946 to July 19 , 2018. A risk-of-bias assessment was conducted for all included studies, and outcomes were pooled using a random effects model. The primary outcome was improvement in pain up to 2 years. Secondary outcomes were functional outcome scores reported in the short term ( $\leq 1 \mathrm{yr}$ ) and long term ( $\geq 2 \mathrm{yr}$ ). Heterogeneity was assessed using the $l^{2}$ statistic. Functional outcome scores were presented along with minimal clinically important differences to provide clinical context for findings.

Results: Thirteen randomized controlled trials ( $n=1062$ patients) were included in this review. Eligible patients had a mean age of 48 (standard deviation \pm 4 ) years and $45 \%$ were men. The pooled treatment effect of surgical intervention for shoulder impingement did not demonstrate any benefit to surgery with respect to pain relief (mean difference $-0.07,95 \% \mathrm{Cl}-0.40$ to 0.26 ) or short-term functional outcomes (standardized mean difference $-0.09,95 \%$ confidence interval $[\mathrm{Cl}]-0.27$ to 0.08 ). Surgical intervention did result in a small statistically significant but clinically unimportant improvement in long-term functional outcomes (standardized mean difference $0.23,95 \% \mathrm{Cl} 0.06$ to 0.41 ).

Interpretation: Evidence suggests surgical intervention has little, if any, benefit for impingement pathology in the middle-aged patient. Further research is required to identify those patients who will reliably benefit from surgical intervention as well as optimal conservative treatment strategies.

houlder pain is one of the most common musculoskeletal complaints among Canadian adults. ${ }^{1}$ In the United States over 18.9 million adults reported chronic shoulder pain, and it is responsible for over 4.5 million primary care visits annually. ${ }^{2-4}$ Shoulder impingement can account for up to $85 \%$ of all shoulder complaints; it negatively affects quality of life, results in substantial disability and impairs physical function. ${ }^{5}$ Impingement pathology not only is responsible for a large health burden but also has a substantial and growing economic impact. ${ }^{6}$ It is estimated that almost 300000 surgical procedures for shoulder pathology including impingement are performed annually in the US alone, with the direct financial burden estimated to be over US\$3 billion annually. ${ }^{6,7}$

Most shoulder impingement is degenerative or chronic in nature. Patients typically endorse pain with overhead tasks and daily activities as well as discomfort at night. Conservative treatment is often initially tried, which includes physiotherapy, subacromial cortisone injection and oral anti-inflammatory medications. When symptoms persist, surgical intervention involves subacromial decompression and débridement. ${ }^{8}$ Surgery may be performed via open or arthroscopic means and surgical intervention for impingement pathology has been increasing exponentially. ${ }^{9}$ Subacromial decompression has become one of the most common orthopedic procedures and it is increasingly performed in a minimally invasive outpatient manner with the use of arthroscopy. Subacromial decompression surgery increased sevenfold from 2000 to 2010 in the United Kingdom and fourfold from 1996 to 2006 in the US. ${ }^{6,10,11}$

Competing interests: Asheesh Bedi reports personal fees from Arthrex outside the submitted work. Olufemi Ayeni reports personal fees from Smith \& Nephew and ConMed outside the submitted work. Mohit Bhandari reports personal fees from AgNovos Healthcare, Sanofi Aventis, Smith \& Nephew and Stryker and grants from DJ Orthopedics and Ferring Pharmaceuticals outside the submitted work. No other competing interests were declared.

This article has been peer reviewed.

Correspondence to: Moin Khan, moinkhanmd@gmail.com CMAJ Open 2019. DOI:10.9778/cmajo.20180179 
Guidelines evaluating evidence for surgery remain inconclusive. ${ }^{12,13}$ Earlier reviews did not include the findings from numerous recent randomized controlled trials. ${ }^{14}$ The objective of this systematic review and meta-analysis was to evaluate the efficacy of surgical intervention in comparison with nonoperative or sham treatments in patients with shoulder impingement in terms of both pain and functional outcomes.

\section{Methods}

This study was conducted according to the methods of the Cochrane handbook ${ }^{15}$ and is reported according to the Preferred Reporting Items for Systematic Reviews and MetaAnalyses (PRISMA) statement. ${ }^{16}$

\section{Eligibility criteria}

The eligibility criteria were as follows: (a) studies that involved patients over 18 years of age with shoulder impingement and (b) randomized controlled trials comparing operative treatment with nonoperative or sham surgical treatment.

No restriction was made regarding publication date, language, presence or absence of co-interventions, type of nonoperative intervention, or length of follow-up. Case reports, case series, prospective and retrospective cohort studies, editorials, reviews and basic science and cadaveric studies were excluded from this review.

\section{Identification of trials}

Multiple strategies were used to identify potential eligible trials. MEDLINE, Embase and the Cochrane databases were systematically searched in OVID from 1946 to July 19, 2018. The search was performed by the primary author (M.K.); a health sciences librarian experienced in the conduct of systematic reviews assisted in developing and performing the search. $\mathrm{MeSH}$ and Emtree headings and subheadings were used in various combinations and supplemented with free text to increase sensitivity (Appendix 1, available at www.cmajopen.ca/ content/7/1/E149/suppl/DC1). The search strategy was adapted in PubMed to search for articles e-published ahead of print. Conference abstracts from recent major annual orthopedic and sports medicine conferences were reviewed. We also consulted with experts in the field, manually reviewed the reference lists of articles that fulfilled the eligibility criteria and used the related articles feature in PubMed.

\section{Screening and assessment of eligibility}

All titles and abstracts were independently screened for eligibility by 2 experienced reviewers (B.A., N.H.) using a piloted electronic database (Microsoft Excel). All discrepancies were resolved by consensus involving discussion with the senior author (M.K.). Duplicate articles were manually excluded. Both reviewers independently reviewed the full text of all studies identified by title and abstract screening to determine final eligibility.

\section{Assessment of risk of bias}

Two reviewers independently performed duplicate outcomespecific assessment of risk of bias using the Cochrane
Collaboration's tool for assessing risk of bias. ${ }^{15}$ The confidence in the estimate of the effect of the intervention for quality of evidence was performed on the basis of the Grading of Recommendations Assessment, Development and Evaluation (GRADE) approach. ${ }^{17}$

\section{Extraction of data}

Data were extracted independently and in duplicate by both reviewers using a piloted electronic data extraction form.

\section{Outcomes}

The primary outcome was related to pain and secondary outcomes were functional scores and postintervention complications. Functional outcomes were measured by various diseasespecific assessment scales and pain was assessed using a visual analogue scale. Pain was reported up to 2 years after surgery. Functional outcomes were dichotomized to short-term $(\leq 1 \mathrm{yr})$ and long-term ( $\geq 2 \mathrm{yr})$ data.

\section{Statistical analysis}

Interobserver agreement for the reviewers' assessments of study eligibility was calculated with Cohen's $\kappa$ coefficient. ${ }^{18}$ Interobserver agreement for assessments of methodological quality was calculated with the intraclass correlation coefficient. The $\kappa$ and intraclass correlation coefficient were calculated using SPSS software (SPSS Inc.).

Outcome instruments that measured similar constructs were summarized using standardized mean differences (SMDs). ${ }^{19}$ The pooled estimate of effect for each outcome was obtained by pooling individual trial SMDs weighted by sample size using the random effects model based on the inverse variance method. ${ }^{19}$ Scores were transformed when required to ensure that higher scores indicated improved function in all cases. ${ }^{19}$ When standard deviations (SDs) were not available, they were calculated using alternative measures or were otherwise estimated from trials within the same comparison with similar scales, outcomes and time periods. ${ }^{19,20}$ Data were extracted from graphical representations when required. Statistical analysis and pooling of results were performed on patients with complete data at follow-up. When studies compared arthroscopic decompression with placebo surgery and nonoperative treatments, placebo and surgical decompression were statistically compared.

SMD values were converted to Constant score values to improve interpretability. The Constant score evaluates shoulder function by assessing both subjective and objective measurements that include pain, activities of daily living, range of motion and strength. ${ }^{19}$ The score is commonly used in the evaluation of rotator cuff pathology. ${ }^{21}$ The minimal important difference is estimated to be 10.4 points for the Constant score for rotator cuff pathology. ${ }^{22}$ A zone of no important difference based on the converted minimal important difference was projected onto the forest plot to aid interpretability.

Outcomes were dichotomized to short-term results $(\leq 12 \mathrm{mo})$ and long-term data ( $\geq 2 \mathrm{yr})$. Sham surgery and nonoperative treatment were pooled given the similar underlying conservative nature of the procedures. Complications were tabulated and presented descriptively. 
Forest plots were created with Review Manager 5.2 (Nordic Cochrane Centre, Cochrane Collaboration).

\section{Evaluation of heterogeneity and sensitivity analyses}

Heterogeneity was quantified using the $\chi^{2}$ test for heterogeneity and the $I^{2}$ statistic. ${ }^{15} I^{2}$ estimates the proportion of total variability between studies due to heterogeneity rather than chance alone. We considered $I^{2}$ less than $25 \%$ to indicate low heterogeneity and $I^{2}$ greater than $75 \%$ to indicate considerable heterogeneity. ${ }^{15} \mathrm{~A}$ priori hypotheses were developed to explore both potential artifactual and real differences of treatment effect across trials. We planned for subgroup analysis on the basis of the specific surgical approach (open v. arthroscopic approaches) to evaluate effect on treatment outcome. Sensitivity analyses were planned for studies to investigate the effects of missing data, comparisons between nonoperative and placebo treatments, open and arthroscopic procedures, crossovers, and those trials at high risk of bias.

\section{Ethics approval}

Because this was a systematic review and meta-analysis of published studies, no ethics approval was required.

\section{Results}

\section{Search results and study characteristics}

The literature search identified 1699 potentially relevant studies. Thirteen randomized controlled trials $(n=1062$ patients) were eligible for inclusion in this review (Figure 1).
One non-English study was identified (in German), which was translated. ${ }^{23}$ The $\kappa$ for overall agreement between reviewers for the final eligibility decision was 0.90 (95\% confidence interval [CI] 0.80 to 1.00 ).

All 13 trials were performed in Europe. One study ${ }^{24}$ involved multiple centres and the remainder were singlecentre trials. All of the included trials evaluated shoulder impingement and excluded rotator cuff tears. Among the 13 studies, we identified 8 individual randomized controlled trials, with the remaining studies including follow-up data of the original patient cohort. Both Beard and colleagues and Paavola and colleagues compared subacromial decompression with (a) shoulder arthroscopy as a sham procedure and (b) no treatment or exercise. ${ }^{24,25}$ (Table 1 ). Six of the trials were published in the last 5 years (range 1993-2018).

Conservative treatment arms involved physiotherapy, no treatment, or exercise, with some trials including subacromial cortisone injection or other medical analgesic treatment. Surgical treatment for impingement consisted primarily of arthroscopic subacromial decompression. Most of the included studies provided measures of pain and functional outcome scores, the most common being the Constant and Neer shoulder scores. Sample sizes ranged from 42 to 313 patients. The mean age of patients was $48(\mathrm{SD} \pm 4) \mathrm{yr}$, and $45 \%$ of them were men.

Loss to follow-up ranged from $4 \%$ to $35.7 \%$. One trial did not report crossover to the surgical treatment arm..$^{23}$ Of the trials that did report crossovers, the percentages ranged from $4 \%$ to $62 \%$ from conservative to surgical treatment, and the mean crossover across trials was $21 \%$. Crossover from

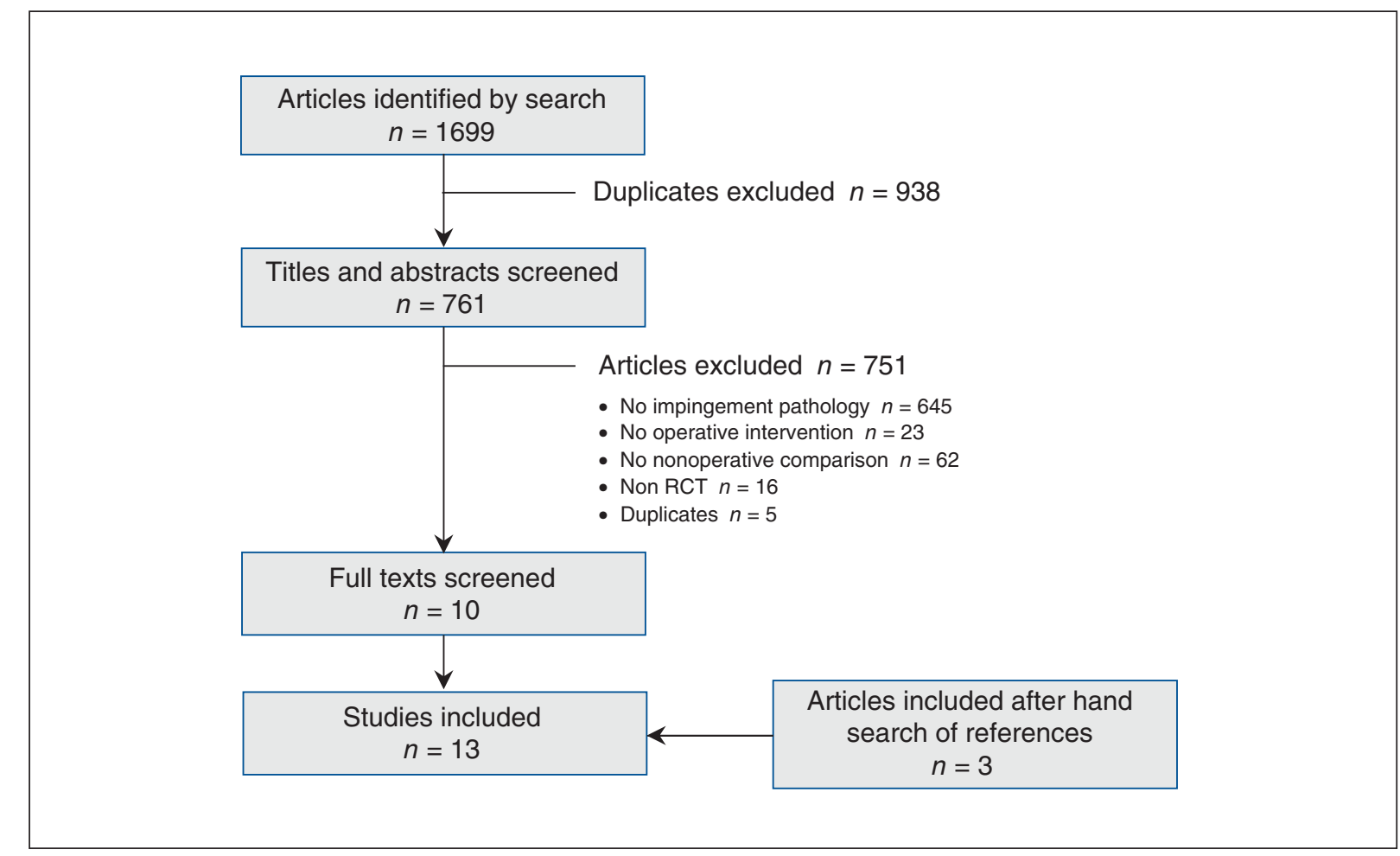

Figure 1: Selection of randomized controlled trials (RCTs) for inclusion in the meta-analysis. 
Table 1 (part 1 of 2): Studies included in the meta-analysis

\begin{tabular}{|c|c|c|c|c|c|c|c|c|c|c|}
\hline \multirow[b]{2}{*}{ Study, year } & \multirow[b]{2}{*}{ Funding } & \multirow[b]{2}{*}{ Country } & \multicolumn{2}{|c|}{$\begin{array}{c}\text { Patient } \\
\text { characteristics }\end{array}$} & \multicolumn{2}{|c|}{$\begin{array}{l}\text { Treatment arm, no. of patients } \\
\text { and description of treatment }{ }^{\star}\end{array}$} & \multirow[b]{2}{*}{ Co-intervention } & \multirow[b]{2}{*}{$\begin{array}{l}\text { Major } \\
\text { outcome } \\
\text { measures }\end{array}$} & \multirow[b]{2}{*}{$\begin{array}{c}\text { Crossover, } \\
\%\end{array}$} & \multirow[b]{2}{*}{$\begin{array}{l}\text { Loss to } \\
\text { follow- } \\
\text { up, } \\
\text { no. }(\%)\end{array}$} \\
\hline & & & $\begin{array}{l}\text { Mean } \\
\text { age, } \\
\text { yr }\end{array}$ & $\begin{array}{l}\text { Male } \\
\text { sex, } \\
\%\end{array}$ & Conservative & Surgical & & & & \\
\hline $\begin{array}{l}\text { Brox et al., } \\
1993^{26}\end{array}$ & $\begin{array}{l}\text { Non- } \\
\text { industry }\end{array}$ & Norway & 48 & 53 & $\begin{array}{l}\text { 50: Supervised } \\
\text { exercises } \\
\text { 30: Placebo } \\
\text { laser }\end{array}$ & $\begin{array}{l}\text { 45: Arthroscopic } \\
\text { decompression }\end{array}$ & $\begin{array}{l}\text { Postop physio } \\
\text { (twice wkly } \\
\text { increasing } \\
\text { resistance } \\
\text { exercises for } \\
3-6 \mathrm{mo} \text { ) }\end{array}$ & $\begin{array}{l}\text { Neer score at } \\
3,6 \text { mo; VAS }\end{array}$ & 4 & $5(4)$ \\
\hline $\begin{array}{l}\text { Peters et al., } \\
1997^{23}\end{array}$ & $\begin{array}{l}\text { Not } \\
\text { stated }\end{array}$ & Germany & 58 & 64 & $\begin{array}{l}\text { 40: } \\
\text { Physiotherapy } \\
\text { plus cortisone } \\
\text { injection }\end{array}$ & $\begin{array}{l}\text { 32: Arthroscopic } \\
\text { or open } \\
\text { decompression }\end{array}$ & Postop physio & $\begin{array}{l}\text { SSRS at } 1 \\
2,3,4 \mathrm{yr}\end{array}$ & NR & NR \\
\hline $\begin{array}{l}\text { Rahme et } \\
\text { al., } 1998^{27}\end{array}$ & $\begin{array}{l}\text { Not } \\
\text { stated }\end{array}$ & Sweden & 42 & 45 & $\begin{array}{l}\text { 21: } \\
\text { Physiotherapy }\end{array}$ & $\begin{array}{l}\text { 21: Open } \\
\text { decompression }\end{array}$ & Postop physio & $\begin{array}{l}\text { VAS at } 6,12 \\
\text { mo }\end{array}$ & 62 & $3(7)$ \\
\hline $\begin{array}{l}\text { Brox et al., } \\
1999^{28}\end{array}$ & $\begin{array}{l}\text { Non- } \\
\text { industry }\end{array}$ & Norway & 48 & 53 & $\begin{array}{l}\text { 50: Supervised } \\
\text { exercises } \\
\text { 30: Placebo } \\
\text { laser }\end{array}$ & $\begin{array}{l}\text { 45: Arthroscopic } \\
\text { decompression }\end{array}$ & $\begin{array}{l}\text { Postop physio } \\
\text { (twice wkly } \\
\text { increasing } \\
\text { resistance } \\
\text { exercises for } \\
3-6 \mathrm{mo} \text { ) }\end{array}$ & $\begin{array}{l}\text { Neer score at } \\
6 \mathrm{mo}, 2.5 \mathrm{yr} ; \\
\text { VAS }\end{array}$ & 33 & $15(12)$ \\
\hline $\begin{array}{l}\text { Haahr et al., } \\
2005^{29}\end{array}$ & $\begin{array}{l}\text { Non- } \\
\text { industry }\end{array}$ & Denmark & 44 & 31 & $\begin{array}{l}\text { 45: } \\
\text { Physiotherapy }\end{array}$ & $\begin{array}{l}\text { 45: Arthroscopic } \\
\text { decompression }\end{array}$ & $\begin{array}{l}\text { Postop physio } \\
\text { (periscapular } \\
\text { muscle and } \\
\text { rotator cuff } \\
\text { strengthening) }\end{array}$ & $\begin{array}{l}\text { Constant } \\
\text { score at 3, } 6, \\
12 \text { mo; PRIM } \\
\text { score }\end{array}$ & 13 & $6(6.7)$ \\
\hline $\begin{array}{l}\text { Haahr et al., } \\
2006^{30}\end{array}$ & $\begin{array}{l}\text { Non- } \\
\text { industry }\end{array}$ & Denmark & 44 & 31 & $\begin{array}{l}\text { 45: } \\
\text { Physiotherapy }\end{array}$ & $\begin{array}{l}\text { 45: Arthroscopic } \\
\text { decompression }\end{array}$ & $\begin{array}{l}\text { Postop physio } \\
\text { (periscapular } \\
\text { muscle and } \\
\text { rotator cuff } \\
\text { strengthening) }\end{array}$ & $\begin{array}{l}\text { PRIM score } \\
\text { at } 4-8 \mathrm{yr}\end{array}$ & 24 & $11(12)$ \\
\hline $\begin{array}{l}\text { Ketola et al., } \\
2009^{31}\end{array}$ & $\begin{array}{l}\text { Not } \\
\text { stated }\end{array}$ & Finland & 47 & 37 & 70: Exercise & $\begin{array}{l}\text { 70: Arthroscopic } \\
\text { decompression }\end{array}$ & $\begin{array}{l}\text { Postop physio } \\
\text { (supervised } \\
\text { therapy with } \\
\text { increasing } \\
\text { resistance for } \\
\text { rotator cuff } \\
\text { strengthening) }\end{array}$ & $\begin{array}{l}\text { SDQ score at } \\
2,5 \text { yr; VAS } \\
\text { score }\end{array}$ & 19.3 & $6(4.2)$ \\
\hline $\begin{array}{l}\text { Ketola et al., } \\
2013^{32}\end{array}$ & $\begin{array}{l}\text { Not } \\
\text { stated }\end{array}$ & Finland & 47 & 37 & 70: Exercise & $\begin{array}{l}\text { 70: Arthroscopic } \\
\text { decompression }\end{array}$ & $\begin{array}{l}\text { Postop physio } \\
\text { (supervised } \\
\text { therapy with } \\
\text { increasing } \\
\text { resistance for } \\
\text { rotator cuff } \\
\text { strengthening) }\end{array}$ & $\begin{array}{l}\text { SDQ score at } \\
2,5 \text { yr; VAS } \\
\text { score }\end{array}$ & 21 & $31(22.1)$ \\
\hline $\begin{array}{l}\text { Farfaras et } \\
\text { al., } 2016^{20}\end{array}$ & $\begin{array}{l}\text { Not } \\
\text { stated }\end{array}$ & Sweden & 50 & 49 & $\begin{array}{l}\text { 34: } \\
\text { Physiotherapy }\end{array}$ & $\begin{array}{l}\text { 29: Arthroscopic } \\
\text { decompression } \\
\text { 24: Open } \\
\text { decompression }\end{array}$ & $\begin{array}{l}\text { Postop physio } \\
\text { (gradual } \\
\text { strengthening } \\
\text { exercises) }\end{array}$ & $\begin{array}{l}\text { Constant } \\
\text { score at 2-3 } \\
\text { yr; SF-36; } \\
\text { Watson and } \\
\text { Sonnebend } \\
\text { score; ROM }\end{array}$ & 16 & $9(10.3)$ \\
\hline $\begin{array}{l}\text { Ketola et al., } \\
2017^{33}\end{array}$ & $\begin{array}{l}\text { Not } \\
\text { stated }\end{array}$ & Finland & 47 & 37 & 70: Exercise & $\begin{array}{l}\text { 70: Arthroscopic } \\
\text { decompression }\end{array}$ & $\begin{array}{l}\text { Postop physio } \\
\text { (supervised } \\
\text { therapy with } \\
\text { increasing } \\
\text { resistance for } \\
\text { rotator cuff } \\
\text { strengthening) }\end{array}$ & $\begin{array}{l}\text { SDQ score at } \\
10 \mathrm{yr} ; \text { VAS }\end{array}$ & 21 & $50(35.7)$ \\
\hline
\end{tabular}


Table 1 (part 2 of 2): Studies included in the meta-analysis

\begin{tabular}{|c|c|c|c|c|c|c|c|c|c|c|}
\hline \multirow[b]{2}{*}{ Study, year } & \multirow[b]{2}{*}{ Funding } & \multirow[b]{2}{*}{ Country } & \multicolumn{2}{|c|}{$\begin{array}{c}\text { Patient } \\
\text { characteristics }\end{array}$} & \multicolumn{2}{|c|}{$\begin{array}{l}\text { Treatment arm, no. of patients }{ }^{*} \\
\text { and description of treatment }\end{array}$} & \multirow[b]{2}{*}{ Co-intervention } & \multirow[b]{2}{*}{$\begin{array}{l}\text { Major } \\
\text { outcome } \\
\text { measures }\end{array}$} & \multirow[b]{2}{*}{$\begin{array}{c}\text { Crossover, } \\
\%\end{array}$} & \multirow{2}{*}{$\begin{array}{l}\text { Loss to } \\
\text { follow- } \\
\text { up, } \\
\text { no. (\%) }\end{array}$} \\
\hline & & & $\begin{array}{l}\text { Mean } \\
\text { age, } \\
\text { yr }\end{array}$ & $\begin{array}{l}\text { Male } \\
\text { sex, } \\
\%\end{array}$ & Conservative & Surgical & & & & \\
\hline $\begin{array}{l}\text { Beard et al., } \\
2018^{24}\end{array}$ & $\begin{array}{l}\text { Non- } \\
\text { industry }\end{array}$ & $\begin{array}{l}\text { United } \\
\text { Kingdom }\end{array}$ & 53 & 50 & $\begin{array}{l}\text { 104: No } \\
\text { treatment } \\
\text { 103: Sham } \\
\text { surgery } \\
\text { (diagnostic } \\
\text { arthroscopy) }\end{array}$ & $\begin{array}{l}\text { 106: Arthroscopic } \\
\text { decompression }\end{array}$ & $\begin{array}{l}\text { Postop physio } \\
\text { (1-4 sessions) }\end{array}$ & $\begin{array}{l}\text { Oxford } \\
\text { shoulder } \\
\text { score at 6,12 } \\
\text { mo; } \\
\text { Constant- } \\
\text { Murley score; } \\
\text { PainDETECT } \\
\text { score; EQ5D; } \\
\text { VAS; HADS }\end{array}$ & 25 & $48(15)$ \\
\hline $\begin{array}{l}\text { Farfaras et } \\
\text { al., } 2018^{34}\end{array}$ & $\begin{array}{l}\text { Not } \\
\text { stated }\end{array}$ & Sweden & 50 & 49 & $\begin{array}{l}\text { 34: } \\
\text { Physiotherapy }\end{array}$ & $\begin{array}{l}\text { 29: Arthroscopic } \\
\text { decompression } \\
\text { 24: Open } \\
\text { decompression }\end{array}$ & $\begin{array}{l}\text { Postop physio } \\
\text { (gradual } \\
\text { strengthening } \\
\text { exercises) }\end{array}$ & $\begin{array}{l}\text { Constant } \\
\text { score at } 10 \\
\text { yr; SF-36; } \\
\text { Watson and } \\
\text { Sonnebend } \\
\text { score; ROM }\end{array}$ & 9 & $13(14.9)$ \\
\hline $\begin{array}{l}\text { Paavola et } \\
\text { al., } 2018^{25}\end{array}$ & $\begin{array}{l}\text { Non- } \\
\text { industry }\end{array}$ & Finland & 50 & 30 & $\begin{array}{l}\text { 71: Supervised } \\
\text { exercise } \\
63: \text { Sham } \\
\text { surgery } \\
\text { (diagnostic } \\
\text { arthroscopy) }\end{array}$ & $\begin{array}{l}\text { 59: Arthroscopic } \\
\text { decompression }\end{array}$ & $\begin{array}{l}\text { Postop physio } \\
\text { (gradual } \\
\text { strengthening } \\
\text { exercises) }\end{array}$ & $\begin{array}{l}\text { VAS; } \\
\text { Constant } \\
\text { score; simple } \\
\text { shoulder test; } \\
\text { 15D }\end{array}$ & 12 & $7(3.6)$ \\
\hline
\end{tabular}

conservative to surgical treatment was due to inadequate relief of symptoms. Ketola and colleagues reported a 9.2\% crossover from surgical to conservative treatment in which patients cancelled surgery. ${ }^{33}$ Six trials documented patients who declined to participate; the primary reported reason for nonparticipation was a strong preference for either surgical or conservative intervention.

\section{Risk of bias}

Overall study quality was moderate (Figure 2). ${ }^{20,23-34} \mathrm{We}$ found 3 trials to be of high quality and they included interventions to blind outcome assessors and investigators where possible. ${ }^{24,25,31-33}$ Agreement between reviewers in the assessment of risk of bias was high (intraclass correlation coefficient 0.92 , $95 \%$ CI 0.86 to 0.95 ). A summary of the findings with respect to outcomes is presented along with an evaluation of the quality of the evidence based on the Grading of Recommendations Assessment, Development and Evaluation (GRADE) approach in Appendix 2 (available at www.cmajopen.ca/ content/7/1/E149/suppl/DC1). ${ }^{17}$

\section{Pain}

Surgical intervention for shoulder impingement did not improve pain at less than 2 years across 6 trials ${ }^{23-26,29,32}$ involving a total of 631 patients (mean difference [MD] $-0.07,95 \%$ $\mathrm{CI}-0.40$ to 0.26$)$ with low heterogeneity $\left(p=0.7, I^{2}=0 \%\right)$. (Figure 3).
Subgroup analysis of only high-quality studies did not change the results of our findings (MD $-0.06,95 \%$ CI -0.52 to 0.39$).{ }^{24,25,32}$ Pooled findings from both of the sham-controlled randomized trials also demonstrated no benefit for arthroscopic decompression in comparison with placebo surgery with respect to pain relief (MD 0.04, $95 \%$ CI -0.48 to 0.55$){ }^{24,25}$ Findings from trials comparing surgery with exercise or physiotherapy also demonstrated a consistent finding of no benefit for surgical intervention over nonoperative treatments (MD -0.15 , 95\% CI -0.57 to 0.28$)$.

\section{Short-term functional outcomes}

Surgical intervention for shoulder impingement did not improve short-term ( $\leq 1 \mathrm{yr}$ ) function across 5 trials $^{23-26,29}$ involving a total of 513 patients (SMD -0.09 , 95\% CI -0.27 to 0.08 ) with low heterogeneity ( $p=0.2, I^{2}=28 \%$ ) (Figure 4). This is equivalent to an estimated Constant-Murley score MD of 1.0 (95\% CI -2.9 to 0.87$)$. This assessment was derived from the Constant score in 3 trials $^{24,25,29}$ and from the Neer score and the Subjective Shoulder Rating Scale (SSRS) in 1 trial each..$^{23,26}$ (Figure 4).

Sensitivity analysis related to open and arthroscopic procedures did not change the review findings with respect to short-term functional outcomes when open procedures were excluded (SMD $-0.10,95 \% \mathrm{CI}-0.28$ to 0.09 ) with moderate heterogeneity $\left(p=0.1, I^{2}=46 \%\right)$. 


\begin{tabular}{|c|c|c|c|c|c|c|}
\hline Study & $\begin{array}{c}\text { Adequate } \\
\text { sequence } \\
\text { generation? }\end{array}$ & $\begin{array}{c}\text { Allocation } \\
\text { concealment? }\end{array}$ & Blinding? & $\begin{array}{l}\text { Incomplete } \\
\text { outcome data } \\
\text { assessed? }\end{array}$ & $\begin{array}{l}\text { Free of } \\
\text { selective } \\
\text { reporting? }\end{array}$ & $\begin{array}{c}\text { Free of other } \\
\text { bias? }\end{array}$ \\
\hline \multicolumn{7}{|l|}{ Beard et al. ${ }^{24}$} \\
\hline \multicolumn{7}{|l|}{ Brox et al. ${ }^{26}$} \\
\hline \multicolumn{7}{|l|}{ Brox et al. ${ }^{28}$} \\
\hline \multicolumn{7}{|l|}{ Farfaras et al. ${ }^{20}$} \\
\hline \multicolumn{7}{|l|}{ Farfaras et al. ${ }^{34}$} \\
\hline \multicolumn{7}{|l|}{ Haahr et al. ${ }^{29}$} \\
\hline \multicolumn{7}{|l|}{ Haahr et al. ${ }^{30}$} \\
\hline \multicolumn{7}{|l|}{ Ketola et al. ${ }^{31}$} \\
\hline \multicolumn{7}{|l|}{ Ketola et al. ${ }^{32}$} \\
\hline \multicolumn{7}{|l|}{ Ketola et al. ${ }^{33}$} \\
\hline \multicolumn{7}{|l|}{ Peters et al. ${ }^{23}$} \\
\hline \multicolumn{7}{|l|}{ Paavola et al. ${ }^{25}$} \\
\hline \multicolumn{7}{|l|}{ Rahme et al. ${ }^{27}$} \\
\hline & & Low & in & High & & \\
\hline
\end{tabular}

Figure 2: Risk-of-bias assessment of randomized controlled trials included in the meta-analysis.

\section{Long-term functional outcomes}

Surgical intervention for rotator cuff disease demonstrated a statistically but clinically unimportant benefit compared with nonoperative treatment in long-term functional outcome ( $\geq 2 \mathrm{yr}$ ) across 6 trials ${ }^{20,23,25,28,30,32}$ involving a total of 507 patients (SMD $0.23,95 \%$ CI 0.06 to $0.41, I^{2}=0 \%$ ). This is equivalent to an estimated Constant-Murley score MD of 2.51 (95\% CI 0.65 to 4.47). This assessment was derived from the Constant score in 2 trials $^{20,25}$ and from the Neer, ${ }^{28}$ Project on Research and Intervention in Monotonous Work (PRIM), ${ }^{30}$ Shoulder Disability Questionnaire $(\mathrm{SDQ})^{32}$ and $\mathrm{SSRS}^{23}$ scores in 1 trial each. This treatment effect did not exceed the threshold of patient importance on the basis of minimal important difference (Figure 5).

\section{Adverse events}

Two trials reported the presence of adverse events. The most commonly reported adverse event was adhesive capsulitis. Beard and colleagues reported 6 cases of adhesive capsulitis, 2 in each of their treatment groups; ${ }^{24}$ Paavola and colleagues reported 2 cases of adhesive capsulitis in patients undergoing exercise treatment, 1 case in those undergoing diagnostic arthroscopy and 3 cases in the subacromial decompression group. ${ }^{25,28}$ There was no significant difference in the odds of adverse events between the groups receiving subacromial decompression and placebo surgery (odds ratio $1.34,95 \%$ CI 0.35 to 5.10 ). ${ }^{24,25}$

\section{Sensitivity analysis}

We conducted a sensitivity analysis to evaluate the effects of estimated missing SDs on long-term function through the 


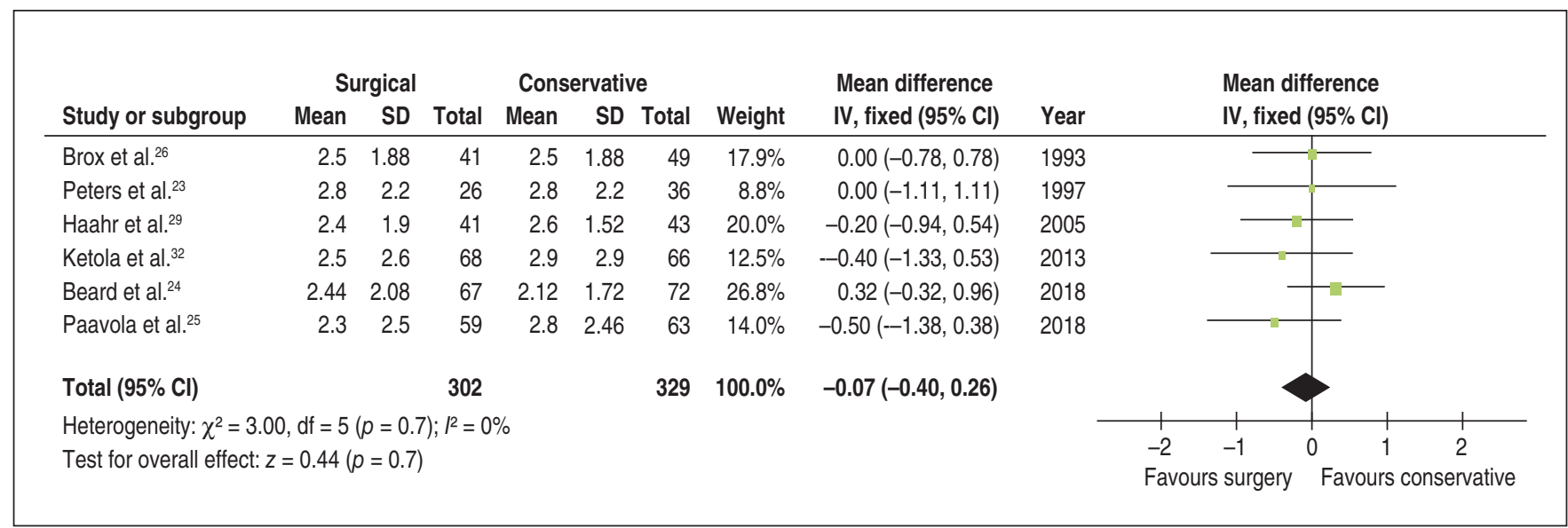

Figure 3: Pooled visual analogue scale pain $(<2 \mathrm{yr})$ outcomes of conservative and surgical treatment for shoulder impingement. Note: $\mathrm{Cl}=$ confidence interval, IV = inverse variance, $\mathrm{SD}=$ standard deviation.

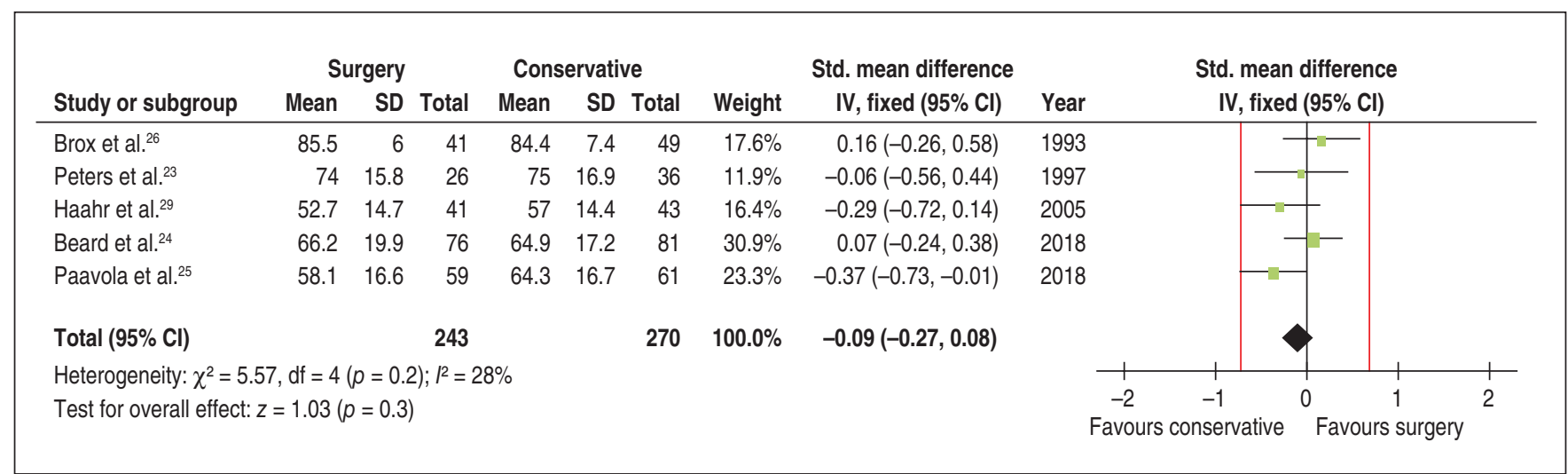

Figure 4: Pooled short-term ( $\leq 1 \mathrm{yr}$ ) functional outcomes of conservative and surgical treatment. Red lines show a zone of no important difference based on a minimal important difference of 10.4 points on the Constant-Murley score. Note: $\mathrm{Cl}=$ confidence interval, IV= inverse variance. $\mathrm{SD}=$ standard deviation, Std. $=$ standardized.

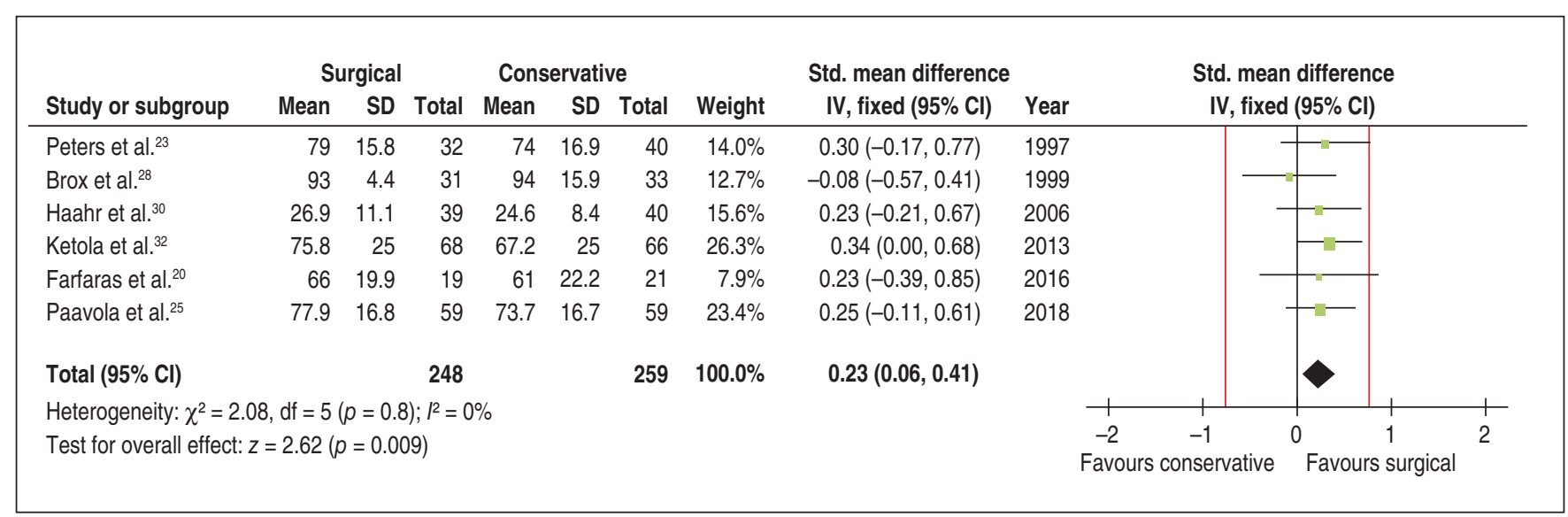

Figure 5: Pooled long-term (> $2 \mathrm{yr}$ ) functional outcomes of conservative and surgical treatment. Red lines show a zone of no important difference based on a minimal important difference of 10.4 points on the Constant-Murley score. Note: $\mathrm{Cl}=$ confidence interval, IV $=$ inverse variance, $\mathrm{SD}=$ standard deviation, Std. $=$ standardized.

removal of studies that required estimated SDs. The results were not significant (SMD $0.24,95 \% \mathrm{CI}-0.02$ to 0.50 ), with no heterogeneity $\left(p=1.00, I^{2}=0 \%\right)$. Similarly, sensitivity analysis with respect to pain outcomes did not change the review findings ( $\mathrm{MD}-0.04,95 \% \mathrm{CI}-0.46$ to 0.38 ) with low heterogeneity ( $\left.p=0.29, I^{2}=19 \%\right)$. Sensitivity analysis on the effect of substantial crossover (>15\%) on the findings did not change the results with respect to long-term function (SMD 
$0.29,95 \%$ CI 0.09 to 0.48 ), short-term function (SMD -0.16, $95 \% \mathrm{CI}-0.37$ to 0.05 ) or pain outcomes (MD $-0.22,95 \% \mathrm{CI}$ -0.60 to 0.17$)$.

\section{Interpretation}

Surgical intervention for subacromial impingement syndrome may have little if any benefit with respect to pain and functional outcomes in the short and long term in comparison with nonoperative treatments such as exercise and physiotherapy alone. Our findings are strengthened by the inclusion of randomized controlled trials, a sufficiently large pooled sample of patients, confidence intervals that excluded our threshold of a minimal clinical importance and low heterogeneity across studies.

The results of this systematic review and meta-analysis, while similar, expand on the findings of Dorrestijn and colleagues and Saltychev and colleagues, who evaluated randomized controlled trials comparing surgical and conservative treatment for subacromial impingement syndrome. ${ }^{35,36}$ Both of those studies pooled results from 4 randomized controlled trials and concluded that the available evidence does not support surgical intervention. However, the previous reviews had substantial limitations. Dorrestijn and colleagues did not perform any statistical pooling of data and Saltychev and colleagues only pooled results for pain, with no assessment of functional outcome. Additionally, both reviews were limited by the fact that they included few trials and did not comment on reported adverse events. The moderate heterogeneity $\left(I^{2}\right.$ $40 \%$ ) in the study by Soltychev must limit confidence in their findings; in contrast, we found no heterogeneity across the studies included in our analysis.

The results of this review identified a small statistically significant benefit for surgical intervention for subacromial impingement in comparison with conservative treatment with respect to improvement in long-term functional outcome. This statistically significant finding did not exceed the minimal important difference. The minimal important difference is the smallest change in a patient-reported outcome or score that informed patients or proxies would perceive as important or valuable enough to warrant a change in management when considering the potential benefits and harms of the intervention. ${ }^{37,28}$ The minimal important difference can vary across a patient population and by disease; therefore, it is critical that the minimal important difference used is appropriate to the specific population of interest. ${ }^{39}$ Kukkonen and colleagues evaluated the minimal important difference of the Constant score used in this review in patients with rotator cuff pathology to be 10.4 points. ${ }^{22}$ Although this tool has limitations it provides the clinician with an aid to evaluate whether statistically significant outcomes will be clinically meaningful. ${ }^{38}$

The use of sham surgical procedures in which both groups of patients undergo surgical treatment controls for the substantial potential for the placebo effect. This effect is based on the expectations of a patient with respect to the perceived effectiveness of a particular treatment. ${ }^{40}$ Both Paavola and colleagues and Beard and colleagues performed sham-controlled trials in which patients in 1 arm were randomly assigned to undergo arthroscopic subacromial decompression and patients in the other arm were randomly assigned to undergo diagnostic arthroscopy. With both groups of patients undergoing surgical intervention the placebo effect is controlled for, and Paavola and colleagues reported that patients undergoing sham surgery were no more likely to guess they underwent a placebo procedure than those undergoing subacromial decompression. ${ }^{25}$ Both trials found no clinically significant differences between patients undergoing surgical decompression or placebo surgery with respect to pain or functional outcome improvement.

Available instruments may not capture or detect clinically significant improvements in outcomes because of measurement error related to limited sensitivity, limited reliability and/or floor and ceiling effects. ${ }^{41}$ Hessler and colleagues reported the minimal detectable change for the Constant score to be 17 and 18 points for subacromial impingement syndrome and rotator cuff tears, respectively, which is greater than the minimal important difference. ${ }^{41}$ Further study is required to reliably assess outcomes for conservative and surgical management of subacromial impingement syndrome.

The primary findings of this review highlight the importance of conservative treatment as the standard of care for shoulder impingement. Given a lack of benefit from surgical treatment, physicians managing this clinical scenario should avoid surgical intervention for subacromial impingement until conservative measures have been exhausted. Recent systematic reviews evaluating conservative treatment options have identified exercise, subacromial cortisone injections and nonsteroidal anti-inflammatory drugs as having benefit when compared with placebo or no treatment in improving pain and functional outcomes. ${ }^{43-45}$

An important finding of this review was the number of patients who crossed over from conservative treatment to surgical intervention by the end of the study period. We found this number to be variable, with a mean of $19 \%$ (range $4 \%$ to $62 \%$ ) across studies. Beard and colleagues reported that a primary limitation of their study was lack of adherence to treatment allocation not only with conservative to surgical crossovers but also with surgical patients declining surgery given improvements in pain while awaiting surgical intervention. ${ }^{24}$ Per-protocol analysis in studies with substantial crossover may be underpowered, but such studies that performed and reported not only intention-to-treat analysis but also per-protocol analysis did not find statistically and/or clinically significant differences between analysis methods. ${ }^{24,46,47}$ Additionally, in cases where patients allocated to arthroscopic decompression or sham surgery reported persistent symptoms and required unblinding to consider reoperation, Paavola and colleagues found no significant differences in the frequency of unblindings between sham and treatment groups.

Patient belief regarding the perceived efficacy of treatment may play an important role in the success of an intervention. Ketola and colleagues found that when patients were informed of their allocated treatment, $65 \%$ in the surgical group and only $28 \%$ in the conservative treatment group felt full recovery would be possible with the intervention assigned. ${ }^{33}$ 
Contreras and colleagues performed a case series of 49 patients and found difficulty in predicting outcomes following conservative treatment with respect to cuff pathology, age, duration of symptoms or hand dominance. ${ }^{48}$ Future research to identify prognostic factors that will enable clinicians to accurately determine which patients will benefit from surgical intervention will expedite care to those who may otherwise fail conservative treatment.

\section{Limitations}

When data were unavailable, we estimated SDs on the basis of similar studies or derived SDs from other provided measures of variance. A sensitivity analysis confirmed that this was unlikely to change the results of our review. Pooled analysis combined various provided outcome measures; however, given their construct similarities and the fact that published data support comparability and correlation, we believe this was reasonable. ${ }^{21,42}$ Crossover between conservative and surgical treatment was common in many trials; however, a sensitivity analysis excluding trials with substantial crossover did not identify any substantial change in the results of our review.

\section{Conclusion}

This systematic review and meta-analysis found moderate evidence to suggest that there may be no clinically significant benefit to surgical intervention for shoulder impingement in comparison with nonoperative treatment for middle-aged patients. Further research is required to identify those patients who will reliably benefit from surgical intervention as well as optimal conservative treatment strategies.

\section{References}

1. Schopflocher D, Taenzer P, Jovey R. The prevalence of chronic pain in Canada. Pain Res Manag 2011;16:445-50.

2. Luime JJ, Koes BW, Hendriksen IJM, et al. Prevalence and incidence of shoulder pain in the general population; a systematic review. Scand 7 Rheumatol 2004;33:73-81.

3. Mather RC, Koenig L, Acevedo D, et al. The societal and economic value of rotator cuff repair. F Bone Foint Surg Am 2013;95:1993-2000.

4. Andersson G; American Academy of Orthopaedic Surgeons. The burden of musculoskeletal diseases in the United States: prevalence, societal and economic cost. Rosemont (IL): American Academy of Orthopaedic Surgeons; 2008.

5. Ostör AJK, Richards CA, Prevost AT, et al. Diagnosis and relation to general health of shoulder disorders presenting to primary care. Rheumatology (Oxford) 2005:44:800-5.

6. Colvin AC, Egorova N, Harrison AK, et al. National trends in rotator cuff repair. 7 Bone foint Surg Am 2012;94:227-33.

7. Beard D, Rees J, Rombach I, et al. The CSAW Study (Can Shoulder Arthroscopy Work?) - a placebo-controlled surgical intervention trial assessing the clinical and cost effectiveness of arthroscopic subacromial decompression for shoulder pain: study protocol for a randomised controlled trial. Trials 2015;16:210.

8. Wolf BR, Dunn WR, Wright RW. Indications for repair of full-thickness rotator cuff tears. Am $\mathcal{F}$ Sports Med 2007;35:1007-16.

9. Thorpe A, Hurworth M, O'Sullivan P, et al. Rising trends in surgery for rotator cuff disease in Western Australia. ANZ J Surg 2016;86:801-4.

10. Judge A, Murphy RJ, Maxwell R, et al. Temporal trends and geographical variation in the use of subacromial decompression and rotator cuff repair of the shoulder in England. Bone foint 7 2014;96-B:70-4.

11. Vitale MA, Arons RR, Hurwitz S, et al. The rising incidence of acromioplasty. 7 Bone Foint Surg Am 2010;92:1842-50.

12. Pedowitz RA, Yamaguchi K, Ahmad CS, et al. American Academy of Orthopaedic Surgeons clinical practice guideline on: optimizing the management of rotator cuff problems. F Bone foint Surg Am 2012;94:163-7.

13. Diercks R, Bron C, Dorrestijn O, et al. Guideline for diagnosis and treatment of subacromial pain syndrome. Acta Orthop 2014;85:314-22.

14. Coghlan JA, Buchbinder R, Green S, et al. Surgery for rotator cuff disease. Cochrane Database Syst Rev 2008;(1):CD005619.
15. Higgins JP. Cocbrane handbook for systematic reviews of interventions. 5th ed. Vol. 5. Chichester (UK): Wiley-Blackwell; 2008.

16. Moher D, Liberati A, Tetzlaff J, et al. Preferred reporting items for systematic reviews and meta-analyses: the PRISMA statement. 7 Clin Epidemiol 2009;62:1006-12.

17. Guyatt G, Oxman $\mathrm{AD}, \mathrm{Akl} \mathrm{EA}$, et al. GRADE guidelines: 1. IntroductionGRADE evidence profiles and summary of findings tables. 7 Clin Epidemiol 2011;64:383-94.

18. Fleiss JL, Levin B, Paik MC. Statistical methods for rates and proportions. 2nd ed. New York: John Wiley and Sons; 1981.

19. Constant CR, Murley AH. A clinical method of functional assessment of the shoulder. Clin Orthop Relat Res 1987; (214):160-4.

20. Farfaras S, Sernert N, Hallström E, et al. Comparison of open acromioplasty, arthroscopic acromioplasty and physiotherapy in patients with subacromial impingement syndrome: a prospective randomised study. Knee Surg Sports Traumatol Artbrosc Off 2016;24:2181-91.

21. Gilbart MK, Gerber C. Comparison of the subjective shoulder value and the Constant score. F Shoulder Elbow Surg 2007;16:717-21.

22. Kukkonen J, Kauko T, Vahlberg T, et al. Investigating minimal clinically important difference for Constant score in patients undergoing rotator cuff surgery. F Shoulder Elbow Surg 2013;22:1650-5.

23. Peters G, Kohn D. Medium-term clinical results after operative and nonoperative treatment of subacromial impingement. Unfallchirurg 1997;100: 623-9.

24. Beard DJ, Rees JL, Cook JA, et al. Arthroscopic subacromial decompression for subacromial shoulder pain (CSAW): a multicentre, pragmatic, parallel group, placebo-controlled, three-group, randomised surgical trial. Lancet 2018;391:329-38.

25. Paavola M, Malmivaara A, Taimela S, et al. Subacromial decompression versus diagnostic arthroscopy for shoulder impingement: randomised, placebo surgery controlled clinical trial. BMF 2018;362:k2860.

26. Brox JI, Staff PH, Ljunggren AE, et al. Arthroscopic surgery compared with supervised exercises in patients with rotator cuff disease (stage II impingement syndrome). BMF 1993;307:899-903.

27. Rahme H, Solem-Bertoft E, Westerberg CE, et al. The subacromial impingement syndrome. A study of results of treatment with special emphasis on predictive factors and pain-generating mechanisms. Scand 7 Rebabil Med 1998;30:253-62.

28. Brox JI, Gjengedal E, Uppheim G, et al. Arthroscopic surgery versus supervised exercises in patients with rotator cuff disease (stage II impingement syndrome): a prospective, randomized, controlled study in 125 patients with a 2 1/2-year follow-up. F Shoulder Elbow Surg 1999;8:102-11.

29. Haahr JP, Østergaard S, Dalsgaard J, et al. Exercises versus arthroscopic decompression in patients with subacromial impingement: a randomised, controlled study in 90 cases with a one year follow up. Ann Rheum Dis 2005;64:760-4.

30. Haahr JP, Andersen JH. Exercises may be as efficient as subacromial decompression in patients with subacromial stage II impingement: 4-8-years' followup in a prospective, randomized study. Scand 7 Rheumatol 2006;35:224-8.

31. Ketola S, Lehtinen J, Arnala I, et al. Does arthroscopic acromioplasty provide any additional value in the treatment of shoulder impingement syndrome?: A two-year randomised controlled trial. F Bone foint Surg Br 2009;91:1326-34.

32. Ketola S, Lehtinen J, Rousi T, et al. No evidence of long-term benefits of arthroscopicacromioplasty in the treatment of shoulder impingement syndrome: five-year results of a randomised controlled trial. Bone foint Res 2013;2:132-9.

33. Ketola S, Lehtinen JT, Arnala I. Arthroscopic decompression not recommended in the treatment of rotator cuff tendinopathy: a final review of a randomised controlled trial at a minimum follow-up of ten years. Bone foint $\mathcal{F}$ 2017;99-B:799-805.

34. Farfaras S, Sernert N, Rostgard Christensen L, et al. Subacromial decompression yields a better clinical outcome than therapy alone: a prospective randomized study of patients with a minimum 10-year follow-up. Am F Sports Med 2018;46:1397-407.

35. Dorrestijn O, Stevens M, Winters JC, et al. Conservative or surgical treatment for subacromial impingement syndrome? A systematic review. F Shoulder Elbow Surg 2009;18:652-60.

36. Saltychev $M$, Äärimaa V, Virolainen $P$, et al. Conservative treatment or surgery for shoulder impingement: systematic review and meta-analysis. Disabil Rehabil 2015;37:1-8.

37. Johnston BC, Ebrahim S, Carrasco-Labra A, et al. Minimally important difference estimates and methods: a protocol. BMF Open 2015;5:e007953.

38. Schünemann HJ, Guyatt GH. Commentary - goodbye M(C)ID! Hello MID, where do you come from? Health Serv Res 2005;40:593-7.

39. Kim JK, Park MG, Shin SJ. What is the minimum clinically important difference in grip strength? Clin Orthop Relat Res 2014;472:2536-41.

40. Dowrick AS, Bhandari M. Ethical issues in the design of randomized trials: to sham or not to sham. F Bone ft Surg Am 2012;94(Suppl 1):7-10.

41. Henseler JF, Kolk A, van der Zwaal P, et al. The minimal detectable change of the Constant score in impingement, full-thickness tears, and massive rotator cuff tears. F Shoulder Elbow Surg 2015;24:376-81.

42. Tingart M, Bäthis H, Lefering R, et al. Constant score and Neer score. A comparison of score results and subjective patient satisfaction [article in German]. Unfallchirurg 2001;104:1048-54. 
43. Steuri R, Sattelmayer M, Elsig S, et al. Effectiveness of conservative interventions including exercise, manual therapy and medical management in adults with shoulder impingement: a systematic review and meta-analysis of RCTs. Br 7 Sports Med 2017;51:1340-7.

44. Hawk C, Minkalis AL, Khorsan R, et al. Systematic review of nondrug, nonsurgical treatment of shoulder conditions. 7 Manipulative Physiol Ther 2017;40:293-319.

45. Shire AR, Stæhr TAB, Overby JB, et al. Specific or general exercise strategy for subacromial impingement syndrome-does it matter? A systematic literature review and meta analysis. BMC Musculoskelet Disord 2017;18:158.

46. Lambers Heerspink FO, van Raay JJAM, Koorevaar RCT, et al. Comparing surgical repair with conservative treatment for degenerative rotator cuff tears: a randomized controlled trial. 7 Shoulder Elbow Surg 2015;24:1274-81.

47. Moosmayer S, Lund G, Seljom US, et al. Tendon repair compared with physiotherapy in the treatment of rotator cuff tears: a randomized controlled study in 103 cases with a five-year follow-up. 7 Bone foint Surg Am 2014:96:1504-14.

48. Contreras F, Brown HC, Marx RG. Predictors of success of corticosteroid injection for the management of rotator cuff disease. HSS 7 2013;9:2-5.
Affiliations: Division of Orthopaedic Surgery, Department of Surgery (Khan, Alolabi, Horner, Ayeni, Bhandari), McMaster University, Hamilton, Ont.; MedSport (Bedi), University of Michigan, Ann Arbor, Mich.; Department of Clinical Epidemiology and Biostatistics (Bhandari), McMaster University, Hamilton, Ont.

Contributors: Moin Khan and Mohit Bhandari conceived the study, and Moin Khan designed it. Moin Khan, Bashar Alolabi and Nolan Horner extracted the data and all authors contributed to the data analysis. Moin Khan drafted the article and all other authors revised it critically for important intellectual content. All authors gave final approval of the version to be published and agreed to act as guarantors of the work.

Supplemental information: For reviewer comments and the original submission of this manuscript, please see www.cmajopen.ca/content/7/1/ E149/suppl/DC1. 